1 Hacettepe Journal of Mathematics and Statistics

h Volume 47 (2) (2018), 207-217

\title{
On the oscillation of higher order fractional difference equations with mixed nonlinearities
}

\author{
Bahaaeldin Abdalla*, Jehad Alzabut ${ }^{\dagger \ddagger}$ and Thabet Abdeljawad ${ }^{\S}$
}

\begin{abstract}
Based on certain mathematical inequalities and Volterra sum equations, we establish oscillation criteria for higher order fractional difference equations with mixed nonlinearities. The problem is addressed for equations involving Riemann-Liouville and Caputo operators. Two examples are constructed to demonstrate the validity of the proposed assumptions. Our results improve those obtained in the previous works.
\end{abstract}

Keywords: Oscillation of solutions; Fractional difference equations; Mixed nonlinearities.

2000 AMS Classification: 26A33, 39A20, 39A10.

Received: 13.10.2016 Accepted: 24.01.2017 Doi : 10.15672/HJMS.2017.458

\section{Introduction}

The problem of oscillation of solutions has been the target of many researchers during the last decades. Indeed, we have witnessed the appearance of many oscillation and non-oscillation results for several types of differential and difference equations; see the monographs $[1,2,3,4]$ and the papers cited therein. The differential and difference equations with mixed nonlinearities, in particular, have been also under extensive investigations in the literature. Such equations often arise in the growth of bacterial populations with competitive species [5]. Several techniques such as Riccati transformation, mathematical inequalities, principle solution and integral averaging method have been employed to establish sufficient conditions for the oscillation of these types of equations $[6,7,8,9,10,11]$.

${ }^{*}$ Department of Mathematics and General Sciences, Prince Sultan University P. O. Box 66833, Riyadh 11586, Saudi Arabia

${ }^{\dagger}$ Department of Mathematics and General Sciences, Prince Sultan University P. O. Box 66833, Riyadh 11586, Saudi Arabia

Email : jalzabut@psu.edu.sa

${ }^{\ddagger}$ Corresponding Author.

${ }^{\S}$ Department of Mathematics and General Sciences, Prince Sultan University P. O. Box 66833, Riyadh 11586, Saudi Arabia 
Due to its widespread applications in science and engineering, on the other hand, the theory of fractional differential equations has become one of the most attractive topics amongst investigators $[12,13,14]$. Recently, the oscillation problem has been systematically addressed for fractional differential equations [15, 16, 17, 18, 19, 20, 21, 22]. For the corresponding fractional difference equations, however, the oscillation problem is rarely considered and still at its first stage of progress. A few results have been reported in this direction $[23,24,25,26,27]$.

In alignment to the recent developments, we shall investigate the oscillation of solutions for fractional difference equations with mixed nonlinearities of forms

$$
\left\{\begin{array}{c}
\nabla_{a(\alpha)-1}^{\alpha} x(t)-p(t) x(t)+\sum_{i=1}^{n} q_{i}(t)|x(t)|^{\lambda_{i}-1} x(t)=v(t), t \in \mathbb{N}_{a(\alpha)+1}, \\
\left.\nabla_{a(\alpha)-1}^{-(m-\alpha)} x(t)\right|_{t=a(\alpha)}=x(a(\alpha))=c, c \in \mathbb{R},
\end{array}\right.
$$

and

$$
\left\{\begin{array}{c}
{ }^{c} \nabla_{a(\alpha)}^{\alpha} x(t)-p(t) x(t)+\sum_{i=1}^{n} q_{i}(t)|x(t)|^{\lambda_{i}-1} x(t)=v(t), t \in \mathbb{N}_{a(\alpha)}, \\
\nabla^{k} x(a(\alpha))=b_{k}, k \in \mathbb{R}, k=0,1,2, \ldots, m-1,
\end{array}\right.
$$

where $m=[\alpha]+1, \alpha>0, p(t), v(t)$ and $q_{i}(t)(1 \leq i \leq n)$ are functions defined from $\mathbb{N}_{a(\alpha)}$ to $\mathbb{R}$ and $\lambda_{i}(1 \leq i \leq n)$ are ratios of odd positive integers with $\lambda_{1}>\ldots>\lambda_{l}>$ $1>\lambda_{l+1}>\ldots>\lambda_{n}$. The set $\mathbb{N}_{a(\alpha)}$ and the fractional operators $\nabla_{a(\alpha)}^{\alpha}$ and ${ }^{c} \nabla_{a(\alpha)}^{\alpha}$ are to be defined in the next section.

By a solution of equation (1.1) ( or (1.2)), we mean a nontrivial sequence $x(t)$ satisfying equation (1.1) (or (1.2)) for $t \in \mathbb{N}_{a(\alpha)+1}$ (or $t \in \mathbb{N}_{a(\alpha)}$ ) and the initial condition at $t=a(\alpha)$. Such a solution is said to be oscillatory if it is neither eventually positive nor eventually negative. Otherwise, it is called non-oscillatory. Equation (1.1) (or (1.2)) is said to be oscillatory if all its solutions are oscillatory.

The paper is organized as follows. Section 2 contains basic notations on nabla operators and three essential lemmas that will be used in the sequel. Section 3 and Section 4 are devoted to the study of oscillation problem for equations (1.1) and (1.2), respectively. To support the main results, we provide examples for each case in Section 5.

\section{Preliminary assertions}

Before proceeding to the main results, we introduce some notations, terminologies and fundamental assertions that will be used throughout the remaining part of the paper. Let $\mathbb{N}$ and $\mathbb{R}$ be the sets of positive integer and real numbers, respectively. Define the set $\mathbb{N}_{a(\alpha)}=\{a(\alpha), a(\alpha)+1, \ldots\}$ where $a(\alpha)=a+m-1$. Let $\rho(t)=t-1$ and $t^{\bar{\alpha}}=\frac{\Gamma(t+\alpha)}{\Gamma(\alpha)}, t \in$ $\mathbb{R}-\{\ldots,-2,-1,0\}$ where $0^{\bar{\alpha}}=0$ and $m=[\alpha]+1$.

2.1. Definition. For $t \in \mathbb{N}_{a(\alpha)+1}, \alpha>0$, and $x$ is defined on $\mathbb{N}_{a}$, the Riemann-Liouville sum operator $\nabla_{a(\alpha)}^{-\alpha}$ is defined by

$$
\nabla_{a(\alpha)}^{-\alpha} x(t)=\frac{1}{\Gamma(\alpha)} \sum_{s=a(\alpha)+1}^{t}(t-\rho(s))^{\overline{\alpha-1}} x(s) .
$$

2.2. Definition. For $t \in \mathbb{N}_{a(\alpha)+1}, \alpha>0$, and $x$ is defined on $\mathbb{N}_{a}$, the Riemann-Liouville difference operator $\nabla_{a(\alpha)}^{\alpha}$ is defined by

$$
\nabla_{a(\alpha)}^{\alpha} x(t)=\nabla^{m} \nabla_{a(\alpha)}^{-(m-\alpha)} x(t)=\frac{\nabla^{m}}{\Gamma(m-\alpha)} \sum_{s=a(\alpha)+1}^{t}(t-\rho(s))^{\overline{m-\alpha-1}} x(s) .
$$


2.3. Definition. For $t \in \mathbb{N}_{a(\alpha)+1}, \alpha>0$, and $x$ is defined on $\mathbb{N}_{a}$, the Caputo difference operator is defined by

$$
{ }^{c} \nabla_{a(\alpha)}^{\alpha} x(t)=\nabla_{a(\alpha)}^{-(m-\alpha)} \nabla^{m} x(t)=\frac{1}{\Gamma(m-\alpha)} \sum_{s=a(\alpha)+1}^{t}(t-\rho(s))^{\overline{\alpha-1}} \nabla^{m} x(s) .
$$

2.4. Definition. For $t \in \mathbb{N}_{a(\alpha)+1}, \alpha>0$, and $x$ is defined on $\mathbb{N}_{a}$, the power rule is defined by

$$
\nabla_{a(\alpha)}^{-\alpha}(t-a(\alpha))^{\bar{\mu}}=\frac{\Gamma(\mu+1)}{\Gamma(\mu+\alpha+1)}(t-a(\alpha))^{\overline{\mu+\alpha}}
$$

In the proofs of the main results, we will make use of the following essential lemmas. Lemma 1, which is a generalization of Lemma 1 in [5], is a straightforward application of linear algebra whereas Lemma 2 is a transformation of Young inequality. Lemma 3 provides brilliant tool that helps dominating certain expressions.

2.5. Lemma. [5] Let $\left(\alpha_{1}, \alpha_{2}, \ldots, \alpha_{n}\right)$ be an $n$-tuple satisfying $\alpha_{1}>\ldots>\alpha_{l}>1>$ $\alpha_{l+1}>\ldots>\alpha_{n}>0$. Then there exists an $n$-tuple $\left(\eta_{1}, \eta_{2}, \ldots, \eta_{n}\right)$ satisfying

$$
\sum_{i=1}^{l} \alpha_{i} \eta_{i}=\sum_{i=l+1}^{n} \alpha_{i} \eta_{i}
$$

with $\sum_{i=1}^{n} \eta_{i}=1$ and $0<\eta_{i}<1$ for $i=1,2, \ldots, n$.

2.6. Lemma. [28] Suppose that $X, Y$ and $U, V$ are nonnegatives. Then each of the following inequalities are valid

$$
\lambda X Y^{\lambda-1}-X^{\lambda} \leq(\lambda-1) Y^{\lambda}, \lambda>1,
$$

and

$$
\mu U V^{\mu-1}-U^{\mu} \geq(\mu-1) V^{\mu}, 0<\mu<1
$$

where equalities hold if and only if $X=Y$ or $U=V$.

2.7. Lemma. [29] (Stirling's formula) For $\epsilon>0$, we have

$$
\lim _{n \rightarrow \infty} \frac{\Gamma(n) n^{\epsilon}}{\Gamma(n+\epsilon)}=1
$$

where $\Gamma$ is the well known gamma function.

For $t \in \mathbb{N}_{a(\alpha)}$, define $f(t)=\left(\frac{1}{t-a(\alpha)}\right)^{1-\alpha}$. It is clear that $f$ is a decreasing function for $t \in \mathbb{N}_{a(\alpha)+1}$. By the help of this fact and the use of Stirling's formula, we observe the following inequalities

$$
\left|t^{1-\alpha}(t-a(\alpha)+1)^{\overline{\alpha-1}} c\right| \leq\left|\left(\frac{t}{t-a(\alpha)}\right)^{1-\alpha} c\right| \leq\left(\frac{T}{T-a(\alpha)}\right)^{1-\alpha}|c|, t \in \mathbb{N}_{T}
$$

and

or

$$
\left|t^{1-\alpha} \sum_{s=a(\alpha)+1}^{T}(t-s+1)^{\overline{\alpha-1}} F(s)\right| \leq t^{1-\alpha} \sum_{s=a(\alpha)+1}^{T}(t-s+1)^{\overline{\alpha-1}}|F(s)|
$$

$$
\begin{aligned}
\lim _{t \rightarrow \infty}\left|t^{1-\alpha} \sum_{s=a(\alpha)+1}^{T}(t-s+1)^{\overline{\alpha-1}} F(s)\right| & \leq \lim _{t \rightarrow \infty} \sum_{s=a(\alpha)+1}^{T}\left(\frac{t}{t-s}\right)^{1-\alpha}|F(s)| \\
& \leq \sum_{s=a(\alpha)+1}^{T}\left(\frac{T}{T-s}\right)^{1-\alpha}|F(s)|, t \in \mathbb{N}_{T} .
\end{aligned}
$$


In view of (2.7) and (2.8), we define

$$
C(T):=\left(\frac{T}{T-a(\alpha)}\right)^{1-\alpha}|c|+\sum_{s=a(\alpha)+1}^{T}\left(\frac{T}{T-s}\right)^{1-\alpha}|F(s)| .
$$

\section{Oscillation criteria for equation (1.1)}

Our first result provides a solution representation for the solutions of equation (1.1). This result was first introduced in [23] so we state it without proof.

3.1. Lemma. [23] Let $x(t)$ be a solution of equation (1.1). Then, $x(t)$ satisfies the following fractional Volterra sum equation for $t \in \mathbb{N}_{a(\alpha)+1}$

$$
x(t)=\frac{(t-a(\alpha)+1)^{\overline{\alpha-1}}}{\Gamma(\alpha)} x(a(\alpha))+\nabla_{a(\alpha)}^{-\alpha}\left[v(t)+p(t) x(t)-\sum_{i=1}^{n} q_{i}(t)|x(t)|^{\lambda_{i}-1} x(t)\right] .
$$

The first main result in this paper is given by the following theorem.

3.2. Theorem. Let

$$
p(t)>0 \text { and } q_{i}(t)\left\{\begin{array}{l}
\geq 0 \text { for } 1 \leq i \leq l \\
\leq 0 \text { for } l+1 \leq i \leq n
\end{array}\right.
$$

If for some constant $K>0$, we have

$$
\liminf _{t \rightarrow \infty} t^{1-\alpha} \sum_{s=a(\alpha)+1}^{t}(t-s+1)^{\overline{\alpha-1}}\left(v(s)+K \sum_{i=1}^{n} p^{\frac{\lambda_{i}}{\lambda_{i}-1}}(s)\left|q_{i}(s)\right|^{\frac{1}{1-\lambda_{i}}}\right)=-\infty
$$

and

$$
\limsup _{t \rightarrow \infty} t^{1-\alpha} \sum_{s=a(\alpha)+1}^{t}(t-s+1)^{\overline{\alpha-1}}\left(v(s)+K \sum_{i=1}^{n} p^{\frac{\lambda_{i}}{\lambda_{i}-1}}(s)\left|q_{i}(s)\right|^{\frac{1}{1-\lambda_{i}}}\right)=\infty
$$

then equation (1.1) is oscillatory.

Proof. Suppose to the contrary that there exists a non-oscillatory solution $x(t)$ of equation (1.1)). Without loss of generality, we may suppose that $x(t)$ is an eventually positive solution of equation (1.1) for $t \geq T$. It follows from equation (3.1) that

$$
\begin{aligned}
x(t) & \leq \frac{(t-a(\alpha)+1)^{\overline{\alpha-1}}}{\Gamma(\alpha)}|c|+\frac{1}{\Gamma(\alpha)} \sum_{s=a(\alpha)+1}^{T}(t-s+1)^{\overline{\alpha-1}}|F(s)| \\
& +\frac{1}{\Gamma(\alpha)} \sum_{s=T+1}^{t}(t-s+1)^{\overline{\alpha-1}} v(s) \\
& +\frac{1}{\Gamma(\alpha)} \sum_{s=T+1}^{t}(t-s+1)^{\overline{\alpha-1}}\left(p(s) x(s)-\sum_{i=1}^{n} q_{i}(s) x^{\lambda_{i}}(s)\right)
\end{aligned}
$$

where $F(s)=v(s)+p(s) x(s)-\sum_{i=1}^{n} q_{i}(s) x^{\lambda_{i}}(s)$. 
For $t \geq T$, we multiply the above inequality by $\Gamma(\alpha) t^{1-\alpha}$ to get

$$
\begin{aligned}
\Gamma(\alpha) t^{1-\alpha} x(t) & \leq C(T)+t^{1-\alpha} \sum_{s=T+1}^{t}(t-s+1)^{\overline{\alpha-1}} v(s) \\
& +t^{1-\alpha} \sum_{s=T+1}^{t}(t-s+1)^{\overline{\alpha-1}}\left[\sum_{i=1}^{l}\left(\lambda_{i} p(s) x(s)-q_{i}(s) x^{\lambda_{i}}(s)\right)\right] \\
& +t^{1-\alpha} \sum_{s=T+1}^{t}(t-s+1)^{\overline{\alpha-1}}\left[\sum_{i=l+1}^{n}\left(-\operatorname{Lp}(s) x(s)-q_{i}(s) x^{\lambda_{i}}(s)\right)\right],
\end{aligned}
$$

where $L:=\frac{\sum_{i=1}^{l} \lambda_{i}-1}{(n-l)}>0$ and $C$ is defined in (2.9). Let $X_{i}=q_{i}^{\frac{1}{\lambda_{i}}}(s) x(s)$ and $Y_{i}=$ $\left(p(s) q_{i}^{\frac{-1}{\lambda_{i}}}(s)\right)^{\frac{1}{\lambda_{i}-1}}, 1 \leq i \leq l$. Then, by using inequality $(2.5)$ of Lemma 2 , we obtain

$$
\begin{aligned}
\lambda_{i} q_{i}^{\frac{1}{\lambda_{i}}}(s) x(s)\left[\left(p(s) q_{i}^{\frac{-1}{\lambda_{i}}}(s)\right)^{\frac{1}{\lambda_{i}-1}}\right]^{\lambda_{i}-1} & -\left(q_{i}^{\frac{1}{\lambda_{i}}}(s) x(s)\right)^{\lambda_{i}} \\
& =\lambda_{i} p(s) x(s)-q_{i}(s) x^{\lambda_{i}}(s) \\
& \leq\left(\lambda_{i}-1\right)\left[\left(p(s) q_{i}^{\frac{-1}{\lambda_{i}}}(s)\right)^{\frac{1}{\lambda_{i}-1}}\right]^{\lambda_{i}} \\
& =\left(\lambda_{i}-1\right) p^{\frac{\lambda_{i}}{\lambda_{i}-1}}(s) q_{i}^{\frac{1}{1-\lambda_{i}}}(s) .
\end{aligned}
$$

Furthermore, if we set $U_{i}=\left|q_{i}(s)\right|^{\frac{1}{\lambda_{i}}}(s) x(s)$ and $V_{i}=\left(\frac{L}{\lambda_{i}} p(s)\left|q_{i}(s)\right|^{\frac{-1}{\lambda_{i}}}\right)^{\frac{1}{\lambda_{i}-1}}, l+1 \leq$ $i \leq n$. Then, by using inequality (2.6) of Lemma 2 , we get

$$
\begin{aligned}
-\lambda_{i}\left|q_{i}(s)\right|^{\frac{1}{\lambda_{i}}} x(s) & {\left[\left(\frac{L}{\lambda_{i}} p(s)\left|q_{i}(s)\right|^{\frac{-1}{\lambda_{i}}}\right)^{\frac{1}{\lambda_{i}-1}}\right]^{\lambda_{i}-1} } \\
+ & \left(\left|q_{i}(s)\right|^{\frac{1}{\lambda_{i}}} x(s)\right)^{\lambda_{i}}=-L p(s) x(s)+\left|q_{i}(s)\right| x^{\lambda_{i}}(s) \\
\leq & \left(1-\lambda_{i}\right)\left[\left(\frac{L}{\lambda_{i}} p(s)\left|q_{i}(s)\right|^{\frac{-1}{\lambda_{i}}}\right)^{\frac{1}{\lambda_{i}-1}}\right]^{\lambda_{i}} \\
& =\left(1-\lambda_{i}\right)\left(\frac{\lambda_{i}}{L}\right)^{\frac{\lambda_{i}}{\lambda_{i}-1}} p^{\frac{\lambda_{i}}{\lambda_{i}-1}}(s)\left|q_{i}(s)\right|^{\frac{1}{1-\lambda_{i}}} .
\end{aligned}
$$

By virtue of relations (3.6) and (3.7), inequality (3.5) becomes

$$
\begin{aligned}
\Gamma(\alpha) t^{1-\alpha} x(t) & \leq C(T)+t^{1-\alpha} \sum_{s=T+1}^{t}(t-s+1)^{\overline{\alpha-1}} v(s) \\
& +t^{1-\alpha} \sum_{s=T+1}^{t}(t-s+1)^{\overline{\alpha-1}} \sum_{i=1}^{l}\left(\lambda_{i}-1\right) p^{\frac{\lambda_{i}}{\lambda_{i}-1}}(s) q_{i}^{\frac{1}{1-\lambda_{i}}}(s) \\
& +t^{1-\alpha} \sum_{s=T+1}^{t}(t-s+1)^{\overline{\alpha-1}} \sum_{i=l+1}^{n}\left(1-\lambda_{i}\right)\left(\frac{\lambda_{i}}{L}\right)^{\frac{\lambda_{i}}{1-\lambda_{i}}} p^{\frac{\lambda_{i}}{\lambda_{i}-1}}(s)\left|q_{i}(s)\right|^{\frac{1}{1-\lambda_{i}}} \\
& \leq C(T)+t^{1-\alpha} \sum_{s=T+1}^{t}(t-s+1)^{\overline{\alpha-1}} v(s) \\
& +t^{1-\alpha} \sum_{s=T+1}^{t}(t-s+1)^{\overline{\alpha-1}} K \sum_{i=1}^{n} p^{\frac{\lambda_{i}}{\lambda_{i}-1}}(s)\left|q_{i}(s)\right|^{\frac{1}{1-\lambda_{i}}}, t \geq T
\end{aligned}
$$


where $K=\max \left\{\lambda_{1}-1, \max _{l+1 \leq i \leq n}\left(1-\lambda_{i}\right)\left(\frac{\lambda_{i}}{L}\right)^{\frac{\lambda_{i}}{1-\lambda_{i}}}\right\}$. Taking the limit inferior of both sides of inequality (3.8) as $t \rightarrow \infty$ and in view of condition (3.3), we get a contradiction to the assumption that $x(t)>0$. In the case $x(t)$ is eventually negative, one can follow similar arguments to prove the validity of condition (3.4). The proof is finished.

In light of Theorem 5 and its proof, one can conclude the following results:

3.3. Corollary. Let $l=n$ in equation (1.1), then $\lambda_{1}>\lambda_{2}>\ldots>\lambda_{n}>1$. Suppose $p(t)>0, q_{i}(t) \geq 0,1 \leq i \leq n$. If (3.3) and (3.4) hold for some constant $K_{1}>0$, then equation (1.1) is oscillatory.

Proof. Suppose to the contrary that there exists a non-oscillatory solution $x(t)$ of equation (1.1)). Without loss of generality, we may suppose that $x(t)$ is an eventually positive solution of equation (1.1) for $t \geq T$. It follows from equation (3.1) that

$$
\begin{aligned}
\Gamma(\alpha) t^{1-\alpha} x(t) & \leq C(T)+t^{1-\alpha} \sum_{s=T+1}^{t}(t-s+1)^{\overline{\alpha-1}} v(s) \\
& +t^{1-\alpha} \sum_{s=T+1}^{t}(t-s+1)^{\overline{\alpha-1}}\left[\sum_{i=1}^{n}\left(\frac{1}{n} p(s) x(s)-q_{i}(s) x^{\lambda_{i}}(s)\right)\right] .
\end{aligned}
$$

For $t \geq T$, if we set $X_{i}=q_{i}^{\frac{1}{\lambda_{i}}}(s) x(s)$ and $Y_{i}=\left(\frac{1}{n \lambda_{i}} p(s) q_{i}^{\frac{-1}{\lambda_{i}}}(s)\right)^{\frac{1}{\lambda_{i}-1}}, 1 \leq i \leq n$. Then by using inequality $(2.5)$, we obtain

$$
\begin{aligned}
\Gamma(\alpha) t^{1-\alpha} x(t) & \leq C(T)+t^{1-\alpha} \sum_{s=T+1}^{t}(t-s+1)^{\overline{\alpha-1}} v(s) \\
& +t^{1-\alpha} \sum_{s=T+1}^{t}(t-s+1)^{\overline{\alpha-1}} K_{1} \sum_{i=1}^{n} p^{\frac{\lambda_{i}}{\lambda_{i}-1}}(s)\left|q_{i}(s)\right|^{\frac{1}{1-\lambda_{i}}}(s), t \geq T,
\end{aligned}
$$

where $K_{1} \geq \frac{\lambda_{1}-1}{n}$. The remaining part of the proof is similar to that of Theorem 5 , so we omit the details.

3.4. Corollary. Let $l=0$ in equation (1.1), then $1>\lambda_{1}>\lambda_{2}>\ldots>\lambda_{n}$. Suppose $p(t)<0, q_{i}(t) \leq 0,1 \leq i \leq n$. If (3.3) and (3.4) hold for some constant $K_{2}>0$, then equation (1.1) is oscillatory.

3.5. Corollary. Let $p(t) \equiv 0$ and $0<l<n$ in equation (1.1). Assume

$$
q_{i}(t)\left\{\begin{array}{l}
\geq 0 \text { for } 1 \leq i \leq l \\
\leq 0 \text { for } l+1 \leq i \leq n .
\end{array}\right.
$$

If there exists a positive function $r(t)$ on $\mathbb{N}_{a(\alpha)}$ such that for some constant $K_{3}>0$, we have

$$
\liminf _{t \rightarrow \infty} t^{1-\alpha} \sum_{s=a(\alpha)+1}^{t}(t-s+1)^{\overline{\alpha-1}}\left(v(s)+K_{3} \sum_{i=1}^{n} r^{\frac{\lambda_{i}}{\lambda_{i}-1}}(s)\left|q_{i}(s)\right|^{\frac{1}{1-\lambda_{i}}}\right)=-\infty
$$

and

$$
\limsup _{t \rightarrow \infty} t^{1-\alpha} \sum_{s=a(\alpha)+1}^{t}(t-s+1)^{\overline{\alpha-1}}\left(v(s)+K_{3} \sum_{i=1}^{n} r^{\frac{\lambda_{i}}{\lambda_{i}-1}}(s)\left|q_{i}(s)\right|^{\frac{1}{1-\lambda_{i}}}\right)=\infty
$$

then equation (1.1)) is oscillatory. 
Proof. For $\lambda_{1}>\lambda_{2}>\ldots>\lambda_{l}>1>\lambda_{l+1}>\ldots>\lambda_{n}$, by Lemma 1, there exists an $n$-tuple $\left(\eta_{1}, \eta_{2}, \ldots, \eta_{n}\right)$ satisfying $\sum_{i=1}^{l} \lambda_{i} \eta_{i}=\sum_{i=l+1}^{n} \lambda_{i} \eta_{i}$. Suppose to the contrary that there exists a non-oscillatory positive solution $x(t)$ for $t \geq T$. It follows from equation (1.1) that

$$
\begin{aligned}
\Gamma(\alpha) t^{1-\alpha} x(t) & \leq C(T)+t^{1-\alpha} \sum_{s=T+1}^{t}(t-s+1)^{\overline{\alpha-1}} v(s) \\
& +t^{1-\alpha} \sum_{s=T+1}^{t}(t-s+1)^{\overline{\alpha-1}}\left[\sum_{i=1}^{l}\left(\lambda_{i} \eta_{i} r(s) x(s)-q_{i}(s) x^{\lambda_{i}}(s)\right)\right] \\
& +t^{1-\alpha} \sum_{s=T+1}^{t}(t-s+1)^{\overline{\alpha-1}}\left[\sum_{i=l+1}^{n}\left(-\lambda_{i} \eta_{i} r(s) x(s)+\left|q_{i}(s)\right| x^{\lambda_{i}}(s)\right)\right] .
\end{aligned}
$$

The remainder of the proof is similar to the proof of Theorem 5 , hence, we omit the details.

3.6. Remark. The results of this section remain valid for fractional difference equations of the form

$$
\left\{\begin{array}{c}
\nabla_{a-1}^{\alpha} x(t)-p(t) x(t)+\sum_{i=1}^{n} q_{i}(t)|x(t)|^{\lambda_{i}-1} x(t)=v(t), t \in \mathbb{N}_{a+1}, \\
\left.\nabla_{a-1}^{-(1-\alpha)} x(t)\right|_{t=a}=x(a)=c, c \in \mathbb{R}
\end{array}\right.
$$

where $m=1$. Indeed, the solution of (3.12) is equivalent to the fractional Volterra sum equation

$$
x(t)=\frac{(t-a+1)^{\overline{\alpha-1}}}{\Gamma(\alpha)} x(a)+\nabla_{a}^{-\alpha}\left[v(t)+p(t) x(t)-\sum_{i=1}^{n} q_{i}(t)|x(t)|^{\lambda_{i}-1} x(t)\right] .
$$

\section{Oscillation criteria for equation (1.2)}

In this section, we establish oscillation criteria for equation (1.2). The results are similar to those provided in Section 3 thus stated without proofs.

4.1. Lemma. [23] Let $x(t)$ be a solution of equation (1.2). Then $x(t)$ satisfies the following fractional Volterra sum equation for $t \in \mathbb{N}_{a(\alpha)}$

$$
x(t)=\sum_{k=0}^{m-1} \frac{(t-a(\alpha))^{\bar{k}}}{k !} b_{k}+\nabla_{a(\alpha)}^{-\alpha}\left[v(t)+p(t) x(t)-\sum_{i=1}^{n} q_{i}(t)|x(t)|^{\lambda_{i}-1} x(t)\right] .
$$

The second main result in this paper is given in the following theorem.

4.2. Theorem. Assume that condition (3.2) holds. If

$$
\liminf _{t \rightarrow \infty} t^{1-m} \sum_{s=a(\alpha)+1}^{t}(t-s+1)^{\overline{\alpha-1}}\left(v(s)+K \sum_{i=1}^{n} p^{\frac{\lambda_{i}}{\lambda_{i}-1}}(s)\left|q_{i}(s)\right|^{\frac{1}{1-\lambda_{i}}}\right)=-\infty
$$

and

$$
\limsup _{t \rightarrow \infty} t^{1-m} \sum_{s=a(\alpha)+1}^{t}(t-s+1)^{\overline{\alpha-1}}\left(v(s)+K \sum_{i=1}^{n} p^{\frac{\lambda_{i}}{\lambda_{i}-1}}(s)\left|q_{i}(s)\right|^{\frac{1}{1-\lambda_{i}}}\right)=\infty
$$

for some constant $K>0$, then equation (1.2) is oscillatory.

4.3. Corollary. Suppose $p(t)>0, q_{i}(t) \geq 0,1 \leq i \leq n$. If (4.1) and (4.2) hold for some constant $K_{1}>0$, then equation (1.2) is oscillatory. 
4.4. Corollary. Suppose $p(t)>0, q_{i}(t) \leq 0,1 \leq i \leq n$. If (4.1) and (4.2) hold for some constant $K_{2}>0$, then equation (1.2) is oscillatory.

4.5. Corollary. Let (3.9) hold. If there exists a positive function $r(t)$ on $\mathbb{N}_{a(\alpha)}$ such that for some constant $K_{3}>0$, we have

$$
\liminf _{t \rightarrow \infty} t^{1-m} \sum_{s=a(\alpha)+1}^{t}(t-s+1)^{\overline{\alpha-1}}\left(v(s)+K_{3} \sum_{i=1}^{n} r^{\frac{\lambda_{i}}{\lambda_{i}-1}}(s)\left|q_{i}(s)\right|^{\frac{1}{1-\lambda_{i}}}\right)=-\infty
$$

and

$$
\limsup _{t \rightarrow \infty} t^{1-m} \sum_{s=a(\alpha)+1}^{t}(t-s+1)^{\overline{\alpha-1}}\left(v(s)+K_{3} \sum_{i=1}^{n} r^{\frac{\lambda_{i}}{\lambda_{i}-1}}(s)\left|q_{i}(s)\right|^{\frac{1}{1-\lambda_{i}}}\right)=\infty
$$

then equation (1.2) is oscillatory.

4.6. Remark. The results of this section are valid for fractional difference equations of the form

$$
\left\{\begin{array}{c}
{ }^{c} \nabla_{a}^{\alpha} x(t)-p(t) x(t)+\sum_{i=1}^{n} q_{i}(t)|x(t)|^{\lambda_{i}-1} x(t)=v(t), t \in \mathbb{N}_{a}, \\
x(a)=b_{0}, b_{0} \in \mathbb{R},
\end{array}\right.
$$

where $m=1$. Indeed, the solution of (4.3) is equivalent to the fractional Volterra sum equation

$$
x(t)=b_{0}+\nabla_{a}^{-\alpha}\left[v(t)+p(t) x(t)-\sum_{i=1}^{n} q_{i}(t)|x(t)|^{\lambda_{i}-1} x(t)\right] .
$$

\section{Examples}

In this section, we construct two examples to illustrate the effectiveness of the assumptions of Theorem 3.2 and Theorem 4.2 .

5.1. Example. Consider the Riemann-Liouville fractional difference equation

$$
\left\{\begin{array}{c}
\nabla_{0}^{\frac{1}{2}} x(t)-t^{2} x(t)+2 t^{3}|x(t)|^{\frac{1}{2}} x(t)-t|x(t)|^{-\frac{1}{2}} x(t)=\cos \pi t, t \in \mathbb{N}_{2}, \\
\left.\nabla_{0}^{-\frac{1}{2}} x(t)\right|_{t=1}=x(1)=1
\end{array}\right.
$$

where $m=1, \alpha=\frac{1}{2}, p(t)=t^{2}, q_{1}(t)=2 t^{3}, q_{2}(t)=-t, \lambda_{1}=\frac{3}{2}, \lambda_{2}=\frac{1}{2}$ and $v(t)=\cos \pi t$. By simple computation, we get $L=\frac{1}{2}$ and $K=\frac{1}{2}$. Moreover, one can figure out that

$\sum_{s=2}^{t}(t-s+1)^{-\frac{1}{2}}\left[\cos \pi s+\frac{1}{2}\left[\left(s^{2}\right)^{3}\left|2 s^{3}\right|^{-2}+\left(s^{2}\right)^{-1}|-s|^{2}\right]\right]=\sum_{s=2}^{t}(t-s+1)^{-\frac{1}{2}}\left(\cos \pi s+\frac{5}{8}\right)$.

However, we observe that

$$
\frac{5}{8} \sum_{s=2}^{t}(t-s+1)^{-\frac{1}{2}}=\frac{5}{4}(t-1)^{\frac{1}{2}}
$$

Therefore, we have

$$
\liminf _{t \rightarrow \infty} t^{\frac{1}{2}}\left(\sum_{s=2}^{t}(t-s+1)^{-\frac{1}{2}}\left(\cos \pi s+\frac{5}{4}(t-s)^{\frac{1}{2}}\right)\right)=-\infty
$$

and

$$
\limsup _{t \rightarrow \infty} t^{\frac{1}{2}}\left(\sum_{s=2}^{t}(t-s+1)^{-\frac{1}{2}}\left(\cos \pi s+\frac{5}{4}(t-s)^{\frac{1}{2}}\right)\right)=\infty .
$$

Thus, by the consequence of Theorem 3.2, equation (5.1) is oscillatory. 
5.2. Example. Consider the following Caputo fractional difference equation

$$
\left\{\begin{array}{c}
{ }^{c} \nabla_{0}^{\frac{3}{2}} x(t)-t^{2} x(t)+2 t^{3}|x(t)|^{\frac{1}{2}} x(t)=\frac{t^{-\frac{1}{2}}}{\Gamma\left(\frac{1}{2}\right)}+2 t^{\frac{9}{2}}-t^{3}, \\
\nabla x(1)=1, \quad x(1)=1,
\end{array}\right.
$$

where $m=2, \alpha=\frac{2}{3}, p(t)=t^{2}, q_{1}(t)=2 t^{3}, \lambda_{1}=\frac{3}{2}$ and $v(t)=\frac{t^{-\frac{1}{2}}}{\Gamma\left(\frac{1}{2}\right)}+2 t^{\frac{9}{2}}-t^{3}$. Noting that $\frac{t^{-\frac{1}{2}}}{\Gamma\left(\frac{1}{2}\right)}+2 t^{\frac{9}{2}}-t^{3} \geq 0$ and employing (2.1) and (2.4), we obtain

$$
\begin{aligned}
t^{1-m} \sum_{s=2}^{t}(t-s+1)^{\overline{\alpha-1}} & \left(v(s)+K p^{\frac{\lambda_{1}}{\lambda_{1}-1}}(s)\left|q_{1}(s)\right|^{\frac{1}{1-\lambda_{1}}}\right) \\
& \geq t^{-1} \sum_{s=2}^{t}(t-s+1)^{\frac{1}{2}}\left(K s^{6} 2^{-2} s^{-6}\right) \\
& =\left(\frac{K}{4}\right) t^{-1} \sum_{s=2}^{t}(t-s+1)^{\frac{1}{2}} \\
& =\left(\frac{K}{4}\right) t^{-1} \Gamma\left(\frac{3}{2}\right) \nabla_{1}^{-\frac{3}{2}}(t-s)^{\overline{0}} \\
& =\left(\frac{K}{6}\right) \frac{\Gamma\left(t+\frac{1}{2}\right)}{t \Gamma(t-1)} .
\end{aligned}
$$

Taking the limit inferior or limit superior of the left hand side of the above inequality, one can easily see that neither condition (4.1) nor condition (4.2) of Theorem 4.2 is satisfied. However, one can also easily verify that $x(t)=t$ is a non-oscillatory solution of (5.2).

\section{Conclusion}

The study of qualitative properties of fractional differential and difference equations has gained considerable attention in the last few years. Readers can obviously observe the tremendous number of papers appearing in the literature which investigate these types of equations. The widespread applications of these equations could be behind the strong motivation that attract researchers' interest. One of the most important properties of these equations is the oscillation of their solutions. In this paper and due to their applications in bacterial population dynamics, in particular, we provide oscillation criteria for solutions of fractional difference equations with mixed nonlinearities. Taking into account the difference between Riemann-Liouville and Caputo operators, the results are carried out for equations involving these two operators. The equations under consideration are given in general form and thus contain some previously considered particular cases. In the proof of the main results, newly established fundamental concepts of discrete fractional calculus have been skillfully employed. At the end of the paper and for numerical treatments, two examples are constructed and investigated. One can easily figure out that the oscillation of equations (5.1) and (5.2) can not be determined by using existing oscillation criteria in the literature. Therefore, the results of this paper are essentially new have their own merits.

\section{Acknowledgement}

The authors would like to thank Prince Sultan University for funding this work through research group Nonlinear Analysis Methods in Applied Mathematics (NAMAM) group number RG-DES-2017-01-17. 


\section{References}

[1] R. P. Agarwal, S. R. Grace, D. O'Regan: Oscillation Theory for Second Order Linear, Half-Linear, Superlinear and Sublinear Dynamic Equations, Kluwer Academic Publishers, 2002.

[2] R. P. Agarwal, M. Bohner, W.-T. Li: Nonoscillation and Oscillation Theory for Functional Differential Equations, Marcel Dekker, New York, 2004.

[3] R. P. Agarwal, M. Bohner, S. R. Grace, D. O'Regan: Discrete Oscillation Theory, Hindawi Publishing Corporation, 2005.

[4] S. H. Saker: Oscillation Theory of Delay Differential and Difference Equations: Second and Third Orders, Verlag Dr. Müller, 2010.

[5] Y. Sun, J. S. W. Wong: Oscillation criteria for second order forced ordinary differential equations with mixed nonlinearities, J. Math. Anal. Appl. 334 (2007), 549-560.

[6] R. P. Agarwal, M. Bohner, W.-S. Cheung, S. R. Grace: Oscillation criteria for forced second order differential equations with mixed nonlinearities, Math. Comput. Modelling 45 (2007), 965-973.

[7] Z. Zhang, X. Wang, H. Han: Oscillation criteria for forced second order differential equations with mixed nonlinearities, Appl. Math. Lett. 22 (7) (2009), 1096-1101.

[8] L. Erbe, T. S. Hassan, A. Peterson: Oscillation of third-order functional dynamic equations with mixed nonlinearities on time scales, J. Appl. math. Comput. 34 (2010), 353-371.

[9] T. S. Hassan, L. Erbe, A. Peterson: Forced oscillation of second order differential equations with mixed nonlinearities, Acta Math. Sci. Ser. B Engl. Ed. 31 (2) (2011). 613-626.

[10] A. Özbekler, A. Zafer: Second order oscillation of mixed nonlinear dynamic equations with several positive and negative coefficients, Duscrete Contin. Dyn. Syst. Suppl. 2011, 11671175.

[11] Z. Xu, A. Cheng: Oscillation of second order differential equations with mixed nonlinearities, Turk J. Math. 38 (2014), 688-705.

[12] S. G. Samko, A. A. Kilbas, O. I. Marichev: Fractional Intergrals and Derivatives: Theory and Applications, Gordon and Beach Science, Yverdon, Switzerland, 1993.

[13] I. Podlubny: Fractional Diffeential Equations, vol. 198 of Mathematics in Science and Engineering, Academiv Press, San Diego, Calif., USA, 1999.

[14] R. Hilfer: Applications of Fractional Calculus in Physics, World Scientific Publishing Company, Singapore, 2000.

[15] S. R. Grace, R. P. Agarwal, P. J. Y. Wong, A. Zafer: On the oscillation of fractional differential equations, Fract. Calc. Appl. Anal. 15 (2) (2012), 222-231.

[16] D.-X. Chen: Oscillation criteria of fractional differential equations, Adv. Difference Equ. 2012, 2012:33.

[17] Q. Feng, F. Meng: Oscillation of solutions to nonlinear forced fractional differential equations, Electon. J. Differential Equations, Vol. 2013 (2013), No. 169, pp. 110.

[18] H. Qin, B. Zheng: Oscillation of a class of fractional differential equations with damping term, The Sci. World Journal Volume 2013, Article ID 685621, 9 pages.

[19] R. Xu: Oscillation criteria for nonlinear fractional differential equations, J. Appl. Math. Volume 2013, Article ID 971357, 7 pages.

[20] J. Yang, A. Liu, T. Liu: Forced oscillation of nonlinear fractional differential equations with damping term, Adv. Difference Equ. (2015) 2015:1.

[21] Y.-Z. Wang, Z.-L. Han, P. Zhao, S.-R. Sun: Oscillation theorems for fractional neutral differential equations, Hacet. J. Math. Stat. 44 (6) (2015), 1477-1488.

[22] Y. Pan, R. Xu: Some new oscillation criteria for a class of nonlinear fractional differential equations, Fract. Diff. Calc. 6 (1) (2016), 17-33.

[23] J. O. Alzabut, T. Abdeljawad: Sufficient conditons for oscillation of nonlinear fractional difference equations, J. Fract. Calc. Appl. 5 (1) (2014), 177-187.

[24] S. Kisalar, M. K. Yıldız, E. Aktopark: Oscillation of higher order fractional nonlinear difference equations, Internat. J. Differ. Equations 10 (2) (2015), 201-212.

[25] B. Abdalla, K. Abudaya, J. Alzabut, T. Abdeljawad: New oscillation criteria for forced nonlinear fractional difference equations, Vietnam J. Math. 45 (2017), 609--618. 
[26] J. Alzabut, T. Abdeljawad, H. Alrabaiah: Oscillation criteria for forced and damped nabla fractional difference equations, J. Comput. Anal. Appl. 24 (8) (2018), 1387-1394.

[27] B. Abdalla: On the oscillation of q-fractional difference equations, Adv. Difference Equ. 2017(1). Article Number: 254.

[28] G. H. Hardy, J. E. Littlewood, G. Polya: Inequalities, Cambridge University Press, Cambridge 1988.

[29] G. E. Andrews, R. Roy: Special Functions, Cambridge University Press, Cambridge 1999. 
